\title{
CMS trigger in Phase-2
}

\author{
Chad Freer ${ }^{* t \ddagger}$ \\ Northeastern University \\ E-mail: chad.freerdcern.ch
}

The High-Luminosity LHC (HL-LHC) will open an unprecedented window on the weak-scale nature of the universe, providing high-precision measurements of the Standard Model as well as searches for new physics beyond the Standard Model. Such studies require information-rich datasets with a statistical power that matches the high luminosity provided by the Phase- 2 upgrade of the LHC. Efficient collection of those datasets will be a challenging task, given the harsh pileup environment with an average of 200 proton-proton interactions per LHC bunch crossing. CMS uses a two level trigger system to select potentially interesting events. The detector readout electronics and DAQ will be upgraded to allow a maximum Level 1 Accept rate of $750 \mathrm{kHz}$, and a latency of $12.5 \mu$ s (or 500 LHC bunch crossings). In addition, the L1 trigger will, for the first time, include tracking information and high-granularity calorimeter information. This paper presents an overview of the changes planned for the CMS trigger in Phase-2.

7th Annual Conference on Large Hadron Collider Physics - LHCP2019

20-25 May, 2019

Puebla, Mexico

\footnotetext{
* Speaker.

${ }^{\dagger}$ Support by the US National Science Foundation under grants PHY-1707666 and PHY-1343486.

${ }^{\ddagger}$ CMS Collaboration
} 


\section{Introduction}

The LHC will remain the most powerful particle accelerator in the world for the next several decades. As such, its full exploitation is integral to the advancement of particle physics as a whole. Part of this exploitation is the extension of the LHC's discovery potential by increasing the luminosity beyond its original design values [1]. In order to attain the increased collision rate required to achieve this increased luminosity the LHC requires major upgrades by the early 2020s as shown in Figure 1.

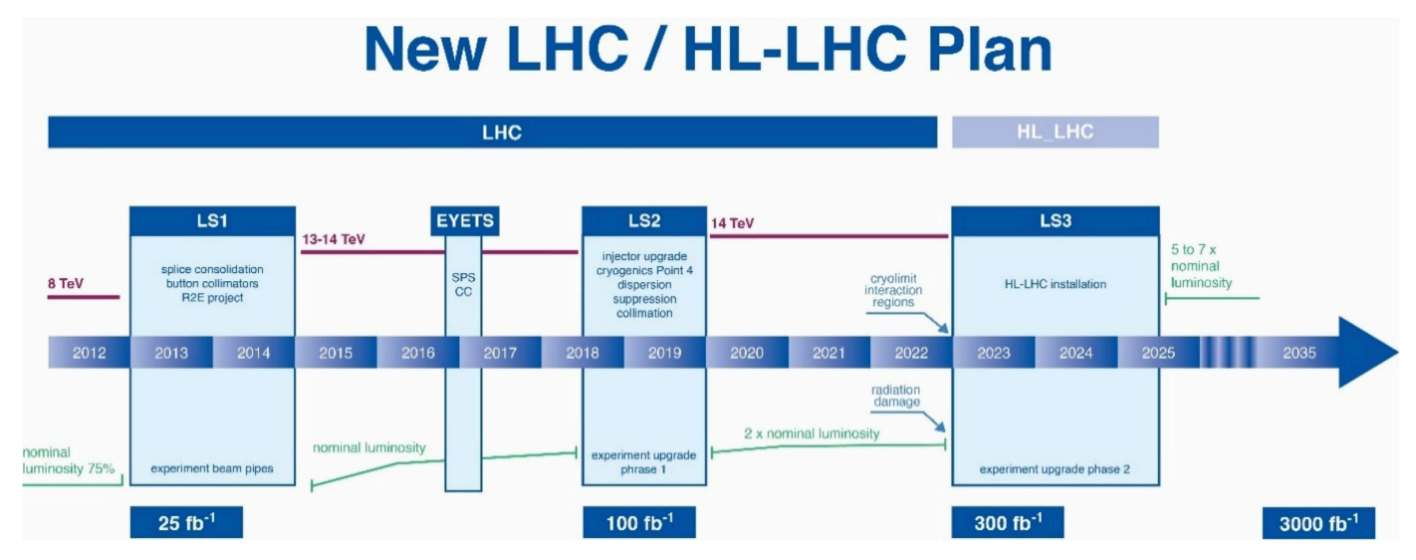

Figure 1: Schedule of the Phase-2 upgrade for the HL-LHC.

Continuing to efficiently collect information-rich datasets will be a challenging task, given the harsh pileup environment of 200 proton-proton interactions per LHC bunch crossing. In order to continue to provide datasets with statistical power matching the high luminosity provided by the Phase-2 upgrade of the LHC, the trigger systems used at the Compact Muon Solenoid (CMS) experiment [2] will need to be upgraded.

The current CMS trigger comprises of two levels; the Level 1 (L1) trigger and the High-Level trigger (HLT) [3]. The L1 trigger is composed of custom hardware processors that receive data from calorimeter and muon systems generating a trigger signal in approximately $3.8 \mu \mathrm{s}$ with a maximum rate of $100 \mathrm{kHz}$ while the HLT is a software based trigger further reducing the rate to 
approximately $1 \mathrm{kHz}$. This two-level strategy will remain through Phase-2, however, the trigger and Data Acquisition (DAQ) systems will be replaced with the goal of increasing the physics reach by expanding the physics menu to a maximum $\mathrm{L} 1$ trigger rate of $750 \mathrm{kHz}(\sim 7.5 \mathrm{kHz}$ HLT rate). Additionally, the L1 trigger will incorporate tracking information and high-granularity calorimeter (HGCAL) information [4] increasing the latency to $12.5 \mu$ s (or 500 LHC bunch crossings). An overview of the Phase-2 trigger system is shown in Figure 2.

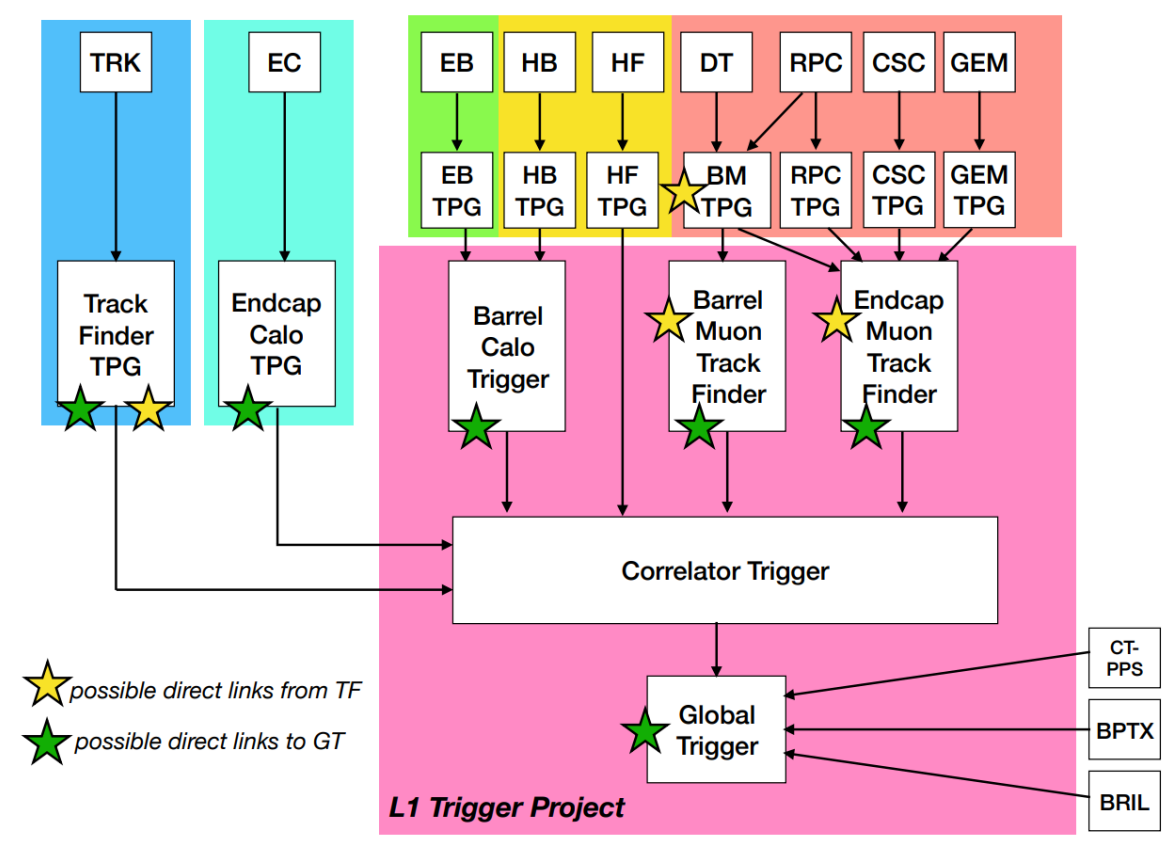

Figure 2: High-level view of the Phase-2 L1 trigger. The main data flow is shown with solid lines. Shown in the diagram are the Outer Tracking Detector (TRK), the Endcap Calorimeter (EC) System, the ECAL Barrel (EB), the HCAL Barrel (HB), the HCAL Forward Detector (HF), the Muon Drift Tube Detectors (DT), the Resistive Plate Chambers (RPC), the Cathode Strip Chambers (CSC), the Gas Electron Multiplier Chambers (GEM). All muon systems are denoted in orange and feed into region specifc muon track finders.

\section{Tracker}

A major new functionality of the trigger for the HL-LHC is the inclusion of the Outer Tracker in the $\mathrm{L} 1$ trigger at a $40 \mathrm{MHz}$ rate. The raw data rate of data from the tracker is enormous but has been reduced by a two step process designed to reduce the data rate caused by soft $p_{T}$ tracks. The first step is a rough local $p_{T}$ measurement using individual $p_{T}$ modules [5]. For each hit in the inner layer of a module, a window corresponding to a high momentum track is opened in the outer 
layer. If a hit is found within this window, a stub containing the position and a rough $p_{T}$ estimation is generated. After the stubs have been generated, tracks are then created from multiple modules giving a more precise $p_{T}$ estimation.

The addition of tracking information $(|\eta|<2.4)$ allows for numerous improvements to the L1 system, such as the ability to add vertex information as well as upgrades to several L1 algorithms. In order to attain these improvements, full track reconstruction is required. At pileup of 200, approximately 15,000 stubs will be sent to the Track Finder (TF) Trigger Primitive Generator (TPG). The TF must be able to reconstruct tracks within approximately $5 \mu$ s including $1 \mu$ s for transmission from the front-end boards. An average of 200 tracks will then be sent to the L1 trigger Correlator Trigger (CT) where trigger objects must be reconstructed and received by the Global Trigger (GT) in another $2.5 \mu \mathrm{s}$. A Level 1 Accept (L1A) is sent back and received by the front-end boards in a further $2 \mu \mathrm{s}$. The target L1 latencies are summarized in Table 1 with a total target L1 latency of $9.5 \mu \mathrm{s}$, well below the design capacity of $12.5 \mu \mathrm{s}$.

\begin{tabular}{|c|c|}
\hline Processing Step & Time $(\mu \mathrm{s})$ \\
\hline Input data received by CT & 5 \\
Trigger objects received by GT & 7.5 \\
L1A received by front-ends & 9.5 \\
\hline
\end{tabular}

Table 1: Targets for L1 Latency.

\section{Calorimeters}

In order to meet the increased trigger latency and rate requirements of the Phase-2 upgrade, several of the calorimeter front-end boards, and in some cases, scintillators will need to be upgraded or replaced. This section describes these changes as well as the replacement of the endcap calorimeters with the HGCAL.

\subsection{Electronic Barrel Calorimters}

The Phase-1 ECAL Barrel (EB) TPG is located on-detector and produces trigger tower sums using 5 X 5 arrays of crystals. Phase- 2 will move the EB TPG to back-end electronics. With this migration off-detector, the baseline EB TP will include a single crystal primitive word greatly increasing the spatial resolution with an option for a cluster primitive word. In either case, the EB TPG will continue to include calibration information, as well as digital filtering of input pulses to calculate the transverse energy $\left(E_{T}\right)$ and timing information.

\subsection{Hadron Barrel and Forward Calorimeters}

The Phase-2 upgrade of the HCAL Barrel (HB) calorimeter replaces the back-end electronics. Additionally, Phase-2 TPs will include depth information from the HCAL[6]. The Phase-2 HB TPG electronics will use the same hardware that is being developed for the EB. 
The hadronic forward (HF) detector will continue operation with Phase-1 front-end and backend electronics. However, in its current configuration the back-end electronics cannot sustain the necessary rate. As such, the replaced back-end boards from the HB will be used in conjunction with the HF back-ends to augment the existing back-end system. This addition will allow the system to maintain the target L1 thresholds while maintaining the same HF TP definition as was used in Phase-1.

\subsection{High Granularity Endcap Calorimeter}

For Phase-2, the endcap calorimeter (EC) will be replaced by the new HGCAL [7]. The HGCAL is a sampling calorimeter using both silicon and scintillator technology for detection. In total there are 28 layers in the electromagnetic section and 24 layers in the hadronic section for a total of 52 layers per endcap. Due to the important bandwidth expected from the detector, only half of electromagnetic layers will be used, however, performance of precise matching between clusters and tracks is maintained.

The Endcap Calorimeter TPG (ECT) is processed in two stages for the HGCAL. The first stage will form two-dimensional (2D) clusters for each layer of HGCAL by summing tower data into a single grid in $\phi$ and $\eta$. The second stage of reconstruction is to form three-dimensional (3D) clusters from multiple layars associating the trigger cell to the closest seed. There is a large amount of data associated with these $3 \mathrm{D}$ clusters but the rate can be significantly reduced with simple $E_{T}$ cuts.

\subsection{L1 Electron and Photon algorithms}

Electron and Photon trigger algorithms use data from both electromagnetic and hadronic calorimeters as well as tracking detectors. The rate and efficiency projections for HL-LHC conditions are shown in Figure 3 for the barrel region. With single crystal granularity projected at Phase-2, the efficiency is near 100 while the rate is also decreased compared to the Phase-1 algorithm. Additionally, with the addition of the tracker information at L1, electron and photon triggers can be separated for the trigger. Projections for these electron and photon specific triggers are shown in Figure 3.

\section{Muon Upgrades}

Similarly to the calorimeter systems, the muon systems in the forward region need to be augmented in order to handle the increased rate and latency required for Phase-2. For the barrel region, in addition to a planned upgrade of the back-end electronics, there has been significant progress on trigger algorithms that have sensitivity to displaced particles. This section describes the upgrades to the endcap region as well as the work being done on increasing the physics sensitivity to long-lived particles. 

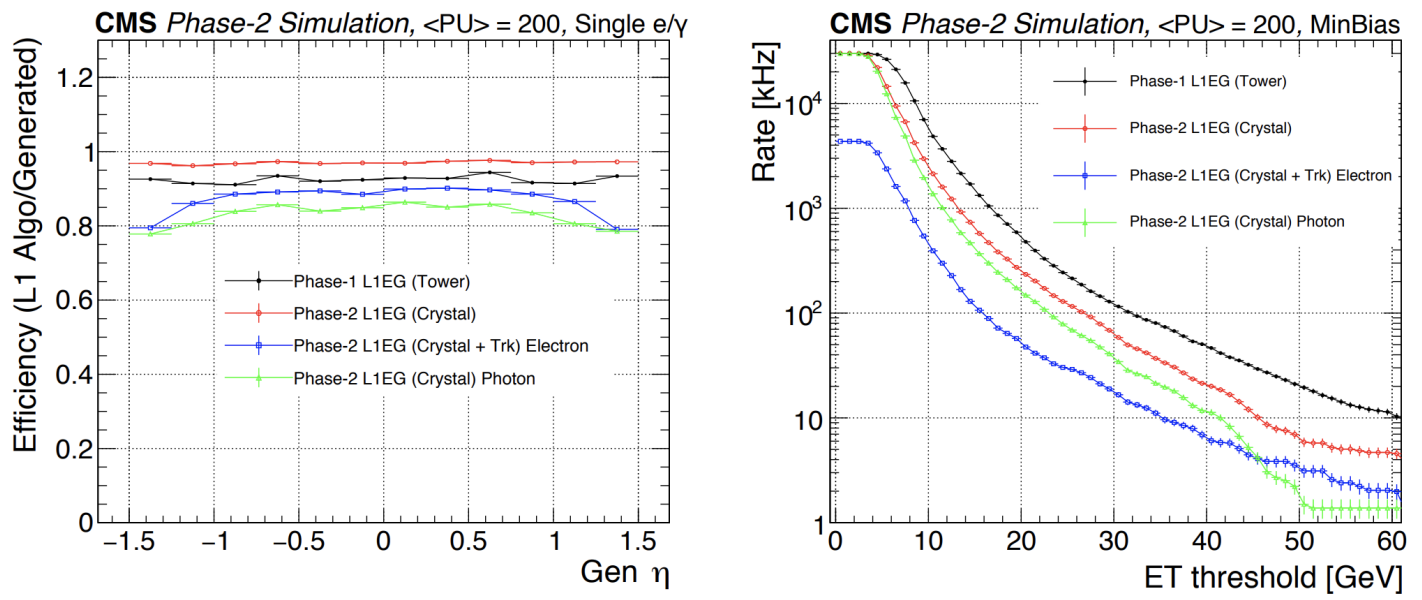

Figure 3: (Left) Expected efficiency of the single electron trigger for the barrel region: calorimeter only, calorimeter photon tuned trigger, and calorimeter matched to the track, compared to the current trigger efficiency as a function of simulated $\eta$ of the electrons/photons for a trigger threshold of $20 \mathrm{GeV}$. (Right) Expected rate for minimum-bias events using the single electron calorimeter trigger (for the barrel region only) as a function of trigger threshold.

\subsection{Endcap Upgrades}

The main change for the endcap region will be the addition of several new detectors to help mitigate the pileup effects expected in this region. The current endcap muon system comprises Cathode strip chambers (CSC) and Resistive Plate Chambers (RPC). By the time of HL-LHC, three additional muon systems will be added; Gas Electron Multiplier (GEM), improved RPC (iRPC) and an additional station (ME0) based on the GEM technology [8]. These additions extend the coverage of the muon system to higher values of $\eta(|\eta|<2.8)$ as well as extra redundancy to mitigate pileup effects. In particular the combination of GEMs and CSCs in the inner station provides greatly increased resolution in the bend angle due to the longer lever arm. The geometry of the system can be seen in Figure 4 while the efficiency and rate with the additional detectors can be seen in Figure 5.

\subsection{Displaced Particle Trigger Algorithms}

Significant effort has been placed on expanding the physics capabilities of the trigger system. Part of this effort has been the development of triggers that are sensitive to displaced particles. The first of these algorithms is the Kalman filter that was fully commissioned in late 2018 for the barrel region. In the Kalman filter algorithm, muon tracks are built from the outer station inwards. This method has shown similar efficiencies and rates as the traditional Phase-1 algorithm but does not constrain the interaction point. The unconstrained vertex greatly increases the efficiency as a function of the transverse impact parameter used in many long-lived searches. 


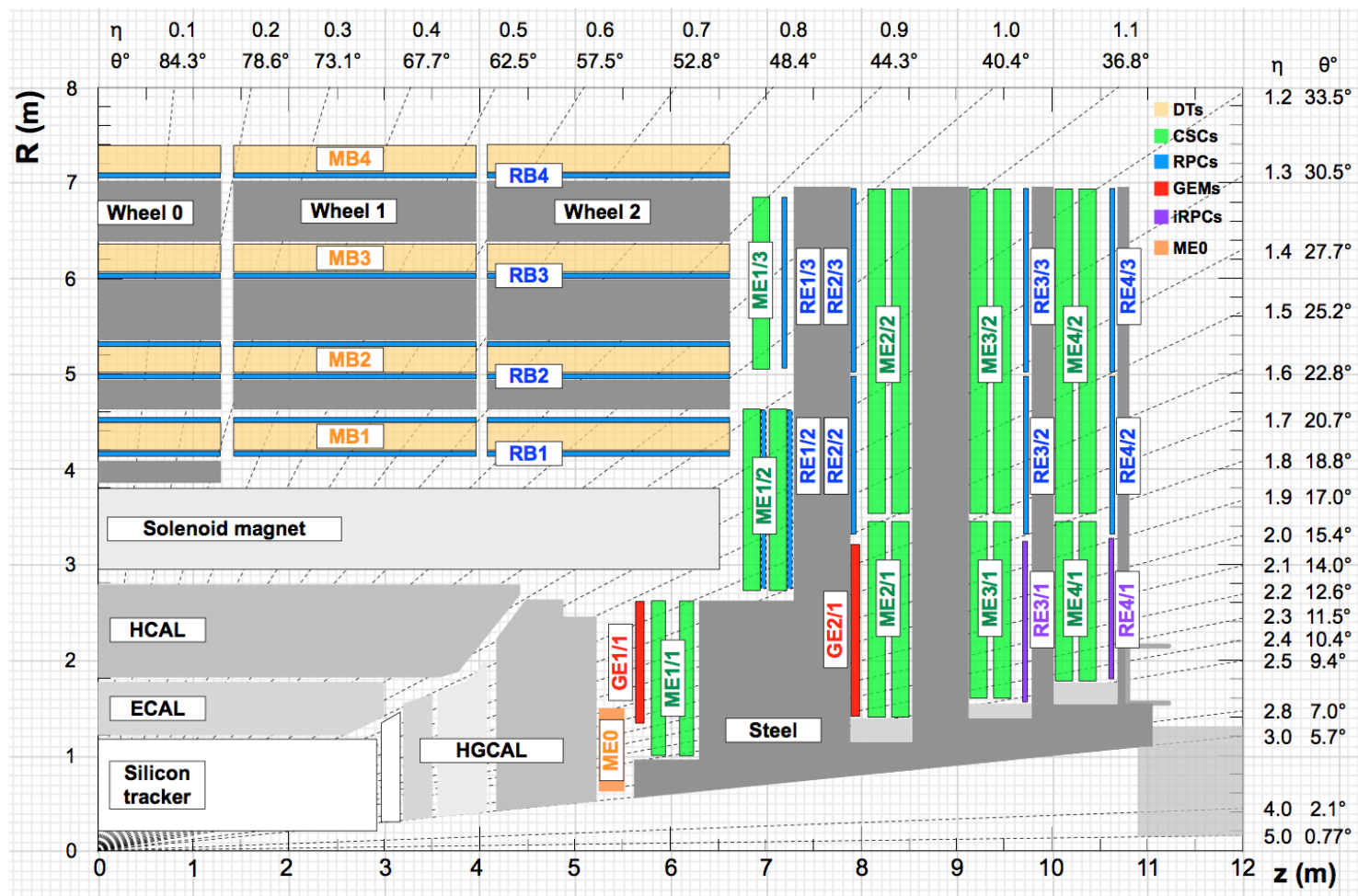

Figure 4: A cross section of a quadrant of the CMS detector, including the Phase-2 upgrades (RE3/1, RE4/1, GE1/1, GE2/1, ME0). The interaction point is at the lower left corner while the magnet yoke is shaded dark grey. The locations of the various muon stations are shown in color $(\mathrm{MB}=$ Drift Tubes, $\mathrm{ME}=\mathrm{CSC}, \mathrm{RB}$ and $\mathrm{RE}=\mathrm{RPC}, \mathrm{GE}$ and $\mathrm{ME} 0=\mathrm{GEM})$.
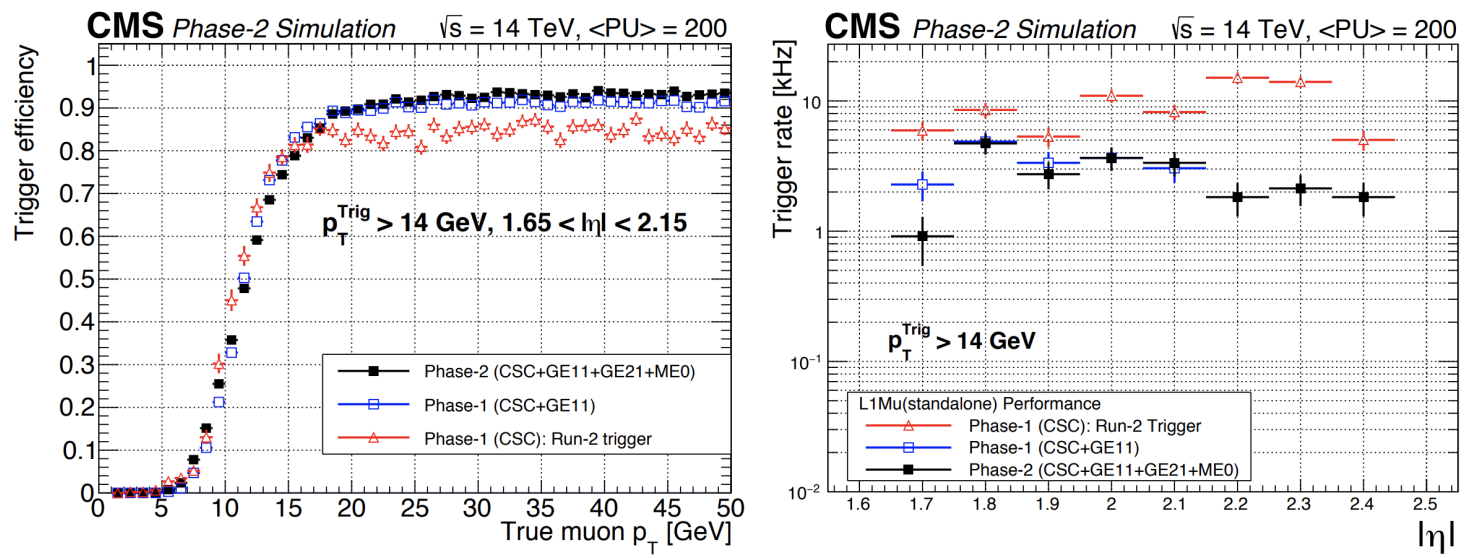

Figure 5: The rate and efficiency comparisons between Phase-1 and Phase-2.

Some theoretical models predict the existence of Heavy Stable Charged Particles (HSCP). These slow moving particles can be identified using time-of-flight measurements from RPC chambers. Phase-2 upgrades of the RPC back-end electronics will increase the timing resolution of hits to aproximately $1.5 \mathrm{~ns}$ to the L1 trigger. This in conjunction with the extended coverage of RPCs 
in the endcap to $|\eta|<2.4$ allows for dedicated HSCP triggers at the L1 level.

\section{Summary and L1 Menu}

Developing in parallel to the implementation of the upgrades listed above is the design of the Phase-2 L1 Menu. For the menu, two scenarios of 140 and 200 pileup are assumed. A simplified menu consisting of 20 major trigger paths that encapsulate the main trigger objects used in physics analyses is considered. The menu shown in Figure 6 covers approximately $70 \%$ of the total L1 Rate that would be needed for a fully operational menu.

Of particular note is the consistency of thresholds between Phase-1 and Phase-2 triggers. Here, the increased rate and pileup at HL-LHC are offset by the upgrades planned for Phase-2, particularly the inclusion of the tracker. With the addition of tracking information at the L1 trigger, L1 Phase-2 algorithms can be made to more closely match algorithms running in the software of the HLT. By combining the complete detector information together (tracking matched to calorimeter and/or muon information), a list of individually reconstructed particle candidates can be created using the CMS particle flow (PF) algorithm [9] significantly increasing the overall CMS trigger performance. This increase in performance corresponds to consistent thresholds for several triggers, in particular the Muon and EGamma triggers, as well as the capability to create future triggers to expand the physics coverage of the L1 Menu.

\section{References}

[1] G Apollinari et al. High-Luminosity Large Hadron Collider (HL-LHC): Preliminary Design Report. CERN Yellow Reports: Monographs. Geneva: CERN, 2015. DOI: 10.5170 /CERN2015-005. URL: http://cds.cern.ch/record/2116337.

[2] S Chatrchyan et al. "The CMS experiment at the CERN LHC. The Compact Muon Solenoid experiment”. In: JINST 3 (2008). Also published by CERN Geneva in 2010, S08004. 361 p. DOI: 10 . 1088/1748-0221/3/08/S08004. URL: https : / cds . cern. ch / record/1129810.

[3] A Tapper and Darin Acosta. CMS Technical Design Report for the Level-1 Trigger Upgrade. Tech. rep. CERN-LHCC-2013-011. CMS-TDR-12. Additional contacts: Jeffrey Spalding, Fermilab, Jeffrey.Spalding@cern.ch Didier Contardo, Universite Claude Bernard-Lyon I, didier.claude.contardo@cern.ch. June 2013. URL: https : / / cds . cern . ch / record / 1556311.

[4] CMS Collaboration. The Phase-2 Upgrade of the CMS L1 Trigger Interim Technical Design Report. Tech. rep. CERN-LHCC-2017-013. CMS-TDR-017. This is the CMS Interim TDR devoted to the upgrade of the CMS L1 trigger in view of the HL-LHC running, as approved by the LHCC. Geneva: CERN, Sept. 2017. URL: https : / / cds . cern . ch / record / 2283192. 


\begin{tabular}{|c|c|c|c|}
\hline $\begin{array}{ll}L=5.6 \times 10^{34} \mathrm{~cm}^{-2} \mathrm{~s}^{-1}, & \langle P U\rangle=140 \\
L=8.0 \times 10^{34} \mathrm{~cm}^{-2} \mathrm{~s}^{-1}, & \langle P U\rangle=200\end{array}$ & \multicolumn{3}{|c|}{$\begin{array}{l}\text { L1 trigger } \\
\text { with L1 tracks }\end{array}$} \\
\hline $\begin{array}{l}\text { Trigger } \\
\text { algorithm }\end{array}$ & \multicolumn{2}{|c|}{$\begin{array}{l}\text { Rate } \\
{[\mathrm{kHz}]}\end{array}$} & $\begin{array}{c}\text { Offline } \\
\text { threshold(s) } \\
{[\mathrm{GeV}]}\end{array}$ \\
\hline$\langle P U\rangle$ & 140 & 200 & \\
\hline Single $\mathrm{Mu}(\mathrm{tk})$ & 14 & 27 & 18 \\
\hline Double Mu (tk) & 1.1 & 1.2 & 1410 \\
\hline $\mathrm{Ele}^{\star}($ iso tk) $+\mathrm{Mu}(\mathrm{tk})$ & 0.7 & 0.2 & 1910.5 \\
\hline Single Ele ${ }^{\star}(\mathrm{tk})$ & 16 & 38 & 31 \\
\hline Single iso $\mathrm{Ele}^{\star}(\mathrm{tk})$ & 13 & 27 & 27 \\
\hline Single $\gamma^{\star}$ (tk-iso) & 31 & 19 & 31 \\
\hline $\mathrm{Ele}^{\star}($ iso tk $)+\mathrm{e} / \gamma^{\star}$ & 11 & 7.3 & 2216 \\
\hline Double $\gamma^{\star}$ (tk-iso) & 17 & 5 & 2216 \\
\hline Single Tau (tk) & 13 & 38 & 88 \\
\hline Tau (tk) + Tau & 32 & 55 & 5656 \\
\hline Ele $^{\star}($ iso tk $)+$ Tau & 7.4 & 23 & 1950 \\
\hline $\mathrm{Tau}(\mathrm{tk})+\mathrm{Mu}(\mathrm{tk})$ & 5.4 & 6 & $45 \quad 14$ \\
\hline Single Jet & 42 & 69 & 173 \\
\hline Double Jet (tk) & 26 & 43 & $2 @ 136$ \\
\hline Quad Jet (tk) & 12 & 45 & $4 @ 72$ \\
\hline Single ele ${ }^{\star}(\mathrm{tk})+$ Jet & 15 & 15 & 2366 \\
\hline Single $\mathrm{Mu}(\mathrm{tk})+$ Jet & 8.8 & 12 & 1666 \\
\hline Single ele $\mathrm{e}^{\star}(\mathrm{tk})+H_{\mathrm{T}}^{\text {miss }}(\mathrm{tk})$ & 10 & 45 & 2395 \\
\hline Single $\mathrm{Mu}(\mathrm{tk})+H_{\mathrm{T}}^{\text {miss }}(\mathrm{tk})$ & 2.7 & 8 & 1695 \\
\hline$H_{\mathrm{T}}(\mathrm{tk})$ & 13 & 24 & 350 \\
\hline Rate for above triggers $^{\star}$ & 180 & 305 & \\
\hline Est. rate (full EG eta range) & & 390 & \\
\hline Est. total $\mathrm{L1}$ menu rate $(\times 1.3)$ & 260 & 500 & \\
\hline
\end{tabular}

Figure 6: Simplified L1 Trigger Menu for Phase-2. Rates are shown for two separate pileup conditions of 140 and 200 with an extra factor of 1.3 for projections to a fully operational L1 Menu. Triggers associated with tracker tracks will be denoted with (tk), triggers isolated away from tracker tracks will be denoted with (tk-iso) while triggers isolated with a track are denoted by (iso tk).

[5] CMS Collaboration. The Phase-2 Upgrade of the CMS Tracker. Tech. rep. CERN-LHCC2017-009. CMS-TDR-014. Geneva: CERN, June 2017. URL: https : / / cds . cern . ch/ record/2272264. 
[6] CMS Collaboration. The Phase-2 Upgrade of the CMS Barrel Calorimeters. Tech. rep. CERNLHCC-2017-011. CMS-TDR-015. This is the final version, approved by the LHCC. Geneva: CERN, Sept. 2017. uRL: https://cds. cern. ch/record/2283187.

[7] CMS Collaboration. The Phase-2 Upgrade of the CMS Endcap Calorimeter. Tech. rep. CERNLHCC-2017-023. CMS-TDR-019. Technical Design Report of the endcap calorimeter for the Phase-2 upgrade of the CMS experiment, in view of the HL-LHC run. Geneva: CERN, Nov. 2017. URL: https: / /cds.cern.ch/record/2293646.

[8] CMS Collaboration. The Phase-2 Upgrade of the CMS Muon Detectors. Tech. rep. CERNLHCC-2017-012. CMS-TDR-016. This is the final version, approved by the LHCC. Geneva: CERN, Sept. 2017. URL: https://cds. cern. ch/record/2283189.

[9] A.M. Sirunyan et al. "Particle-flow reconstruction and global event description with the CMS detector. Particle-flow reconstruction and global event description with the CMS detector". In: JINST 12.CMS-PRF-14-001. CMS-PRF-14-001-004. 10 (June 2017). Replaced with the published version. Added the journal reference and DOI. All the figures and tables can be found at http://cms-results.web.cern.ch/cms-results/public-results/publications/PRF-14-001 (CMS Public Pages), P10003. 82 p. DOI: 10.1088/1748-0221/12/10/P10003. URL: http: //cds.cern.ch/record/2270046. 